\title{
Microcomunidades académicas de conocimiento: el núcleo agropecuario de la Universidad del Zulia
}

\author{
Navarro de G, Ketty* \\ Colina, Belinda** \\ Sepúlveda, Judith ${ }^{\star * *}$
}

\section{Resumen}

El presente artículo es producto de una investigación efectuada en el Núcleo Agropecuario de la Universidad del Zulia (LUZ, Maracaibo, Venezuela), con la finalidad de determinar los factores condicionantes en la conformación de microcomunidades académicas de conocimiento en el Núcleo Agropecuario de LUZ. Se realizó un estudio de tipo exploratorio-descriptivo y su diseño es No experimental-transeccional. Los objetivos específicos son: 1) Explorar las distintas formas de organización de la actividad investigativa que existen en el Núcleo Agropecuario de LUZ; 2) Caracterizar las distintas modalidades de organización del proceso de generación de conocimiento en el Núcleo Agropecuario de la Universidad del Zulia y 3) Determinar la Cultura Organizacional de las Microcomunidades Académicas de Conocimiento del Núcleo Agropecuario de LUZ. Se aplicaron técnicas de investigación cualitativa y cuantitativa. Entre los hallazgos se encontraron evidencias para la construcción de una tipología de formas de organización del proceso de creación académica de conocimiento. Pudiendo identificar tres tipos: Tipo I: Microcomunidades Académicas de Conocimiento. Tipo II: Equipos de Investigación. Tipo III. Grupos de investigación.

Palabras clave: Cultura del conocimiento, gerencia académica, gestión del conocimiento, grupos de investigación, microcomunidades académicas de conocimiento.

Recibido: 28-03-05. Aceptado: 17-09-07

* $\quad$ Lic. en Sociología. Egresada de la Maestría en Planificación y Gerencia de Ciencia y Tecnología, Facultad Experimental de Ciencias de la Universidad del Zulia. Actualmente cursando el doctorado en Creación de Empresas, Dirección de PyMES y Empresas Familiares. Universidad de Castilla, La Mancha, España. Autor para la correspondencia. KettyAsuncion.Navarro1@alu;uclm.es. kettydeg@cantv.net.

** Socióloga. Egresada de la Maestría en Gerencia Pública. Actualmente cursando el doctorado en Ciencias Sociales - Mención Gerencia. Profesora adscrita al Departamento de Ciencias Humanas y a la Maestría en Planificación y Gerencia de Ciencia y Tecnología de la Facultad Experimental de Ciencias de LUZ. belicolina@gmail.com, belindaelena@cantv.net

*** Lic. en Química. Magíster en Ingeniería Ambiental, asistente de proyectos de investigación. 


\section{Academic Micro-Communities of Knowledge: The Agriculture Nucleus at the University of Zulia}

\section{Abstract}

This study is the product of an investigation made at the Agricultural Nucleus in the University of Zulia (LUZ, Maracaibo, Venezuela), to determine the conditioning factors in the conformation of academic micro-communities of knowledge in the Agricultural Nucleus at LUZ. An exploratorydescriptive type study was carried out using a non-experimental - transectional design. Specific objectives were: 1) To explore different forms for organizing research activity in the Agricultural Nucleus of LUZ; 2) To characterize different modes of organizing the knowledge generation process at the Agricultural Nucleus of the University of Zulia; and 3) To determine the organizational culture of academic micro-communities of knowledge in the Agricultural Nucleus at LUZ. Techniques of qualitative and quantitative research were applied. Among the findings, evidences were found for constructing a typology of organizational forms in the academic knowledge creation process. It was possible to identify three types: Type I: Academic Micro-communities of Knowledge; Type II: Research Teams; and Type III: Research Groups.

Key words: Culture of knowledge, academic management, knowledge management, research groups, academic micro-communities of knowledge.

\section{Introducción}

El auge de la sociedad del conocimiento o en otras palabras, la sociedad del aprendizaje permanente, mediante la cual el conocimiento se convierte en uno de los bienes intangibles más preciados de toda organización, significa una oportunidad para recobrar y repotenciar el valor de las universidades tradicionales autónomas, como los reservorios de conocimiento por excelencia; y representa además una vía legitima para el desarrollo de la humanidad. "Así, se afirma que en el paradigma económico de hoy, en el cual la incertidumbre es la única certeza, una fuente segura y duradera de ventaja competitiva es el conocimiento" (Navarro et al., 2006: 266). Ahora más que nunca la generación, organización y difusión de conocimientos han pasado a ser los temas, las preocupaciones privilegiadas e invalorables recursos para el logro de altos niveles de productividad y competitividad, más que cualquier otro bien que tradicionalmente se había considerado; por ello se hace imperioso gerenciarlo.

Más aún lo que realmente es susceptible de ser gerenciada es la organización que genera conocimientos a fin de hacer de ella un entorno propicio para tal fin. Por ello, las empresas más vanguardistas, los empresarios más emprendedores e innovadores en el contexto mundial han adoptado prácticas propias de la gerencia del conocimiento, a fin de hacer de sus organizaciones verdaderos emblemas de progreso. Sin embargo, la gerencia de conocimiento como actividad que busca potenciar, organizar y globalizar el conocimiento no es del interés y uso exclusivo de las empresas. Antes por el contrario, son las universidades las instituciones que tradicional y legítimamente, dada su naturaleza, deben tener ma- 
yor ingerencia en esta materia. "Son las universidades e instituciones de educación superior donde se forma el capital intelectual, que se apropia de los conocimientos complejos que se requieren para desarrollar de igual modo capacidades y competencias complejas en las personas" (Colina, 2007: 48) sin los cuales la ciencia se detendría.

Desde el punto de vista institucional se advierte el surgimiento de universidades corporativas que compiten con las tradicionales, observándose la invasión de terrenos antes del dominio exclusivo de las mismas. Este nuevo estilo de creación y utilización del conocimiento representa una pérdida de espacios, que al parecer las universidades autónomas públicas no han podido o no han sabido evitar, e involucra además para las universidades, el enorme riesgo de perder cada vez más su liderazgo como institución del saber y su rol de institución con una insustituible responsabilidad social.

Especialmente, en Venezuela se argumenta desde hace algún tiempo que las universidades públicas autónomas no disponen de las condiciones gerenciales y organizacionales mínimas para enfrentar los retos que implica la adopción de un modelo gerencial basado en el conocimiento. Sin embargo, la implantación de este modelo gerencial en el ámbito académico, pasa por considerar elementos y factores inherentes a su particularidad, los cuales hacen de esa tarea un proceso mucho más complejo e intrincado. Apoyado en estos argumentos, en la Universidad del Zulia, desde el año 1999 se está desarrollando un proceso de transformación académico-administrativa, basado en una estructura de tipo matricial, con el propósito de asumir la gerencia del conocimiento como práctica gerencial fundamental, para responder con mayor dinamismo a los retos asignados por la sociedad de hoy. No obstante, es preciso señalar que los cambios generados por la vía de decretos se tornan mucho más difíciles de lograr. Uno de los modos de generar cambios en la forma de organización de los procesos generadores de conocimiento, es a través de la evolución progresiva. Otra manera mucho más lenta, no obstante, más segura ocurre por la vía de la inducción. Como parte de esta última emerge el proceso de cambio de los valores inmersos en la cultura.

En atención al anterior planteamiento, Granell (2000) advierte que cuando una organización decide gerenciar el conocimiento, es necesario explorar si cuenta con el conjunto de valores, creencias y conductas organizacionales adecuadas para promover, incentivar y recompensar la innovación, la creatividad, el trabajo en equipo, la toma de riesgos y la capacidad y disposición para compartir la información, la experiencia y el saber; no obstante se puede afirmar que esta exploración aún no se ha iniciado en los distintos organismos de generación de conocimiento existentes en la Universidad del Zulia.

En este artículo se presentan los resultados de una investigación destinada a determinar y analizar los factores condicionantes, para la conformación de microcomunidades académicas de conocimiento en el Núcleo Agropecuario de LUZ. Dichos resultados representan, entre otras cosas, una contribución a fin de desentrañar aquellos valores, motivaciones y otros factores que permiten a los 
grupos de investigación-los cuales constituyen el núcleo central donde se crean los conocimientos que las universidades aportan a la sociedad-avanzar en su empeño, aún sin contar con las condiciones óptimas para la generación de conocimiento. Para ello se estudiaron ocho grupos de investigación de reconocido prestigio y trayectoria en el ámbito universitario, a saber: el Grupo de Investigadores de la Reproducción Animal en la Región Zuliana (GIRARZ), el Grupo de Tecnología de los Alimentos, el Grupo de Diagnóstico de la Unidad de Investigación Clínica de la Facultad de Ciencias Veterinarias, el Grupo de Frutales, el Grupo del Museo de Artrópodos, el Grupo de Ciencia y Tecnología de la Carne, el Programa de Investigación en Comunidades Agrícolas y el Grupo Sistemas de Información de Ganadería de Doble Propósito, todos pertenecientes al núcleo agropecuario de la Universidad del Zulia.

A fin de cumplir con los objetivos trazados se realizó un diseño No Experimental de tipo transeccional, realizando una combinación de tipo exploratorio, descriptivo y explicativo. El estudio efectuado a los grupos de investigación seleccionados fue de dos vertientes: por un lado, se estableció una caracterización de los tipos de organización del trabajo investigativo existentes en el Núcleo Agropecuario de LUZ, resultando de ello un intento por establecer una tipología básica. Por otro lado, se realizó una caracterización al interior de estas microcomunidades académicas de conocimiento, que aporta datos sobre cómo se produce el conocimiento en sus diferentes facetas. Para la primera vertiente se realizó un estudio de carácter exploratorio de tipo cuanti-cualitativo y para el segundo, un estudio descriptivo de tipo cualitativo, el cual permitió desentrañar los conocimientos tácitos que soportan el proceso de creación del conocimiento de los grupos de investigación del Núcleo Agropecuario de LUZ, vistos desde el punto de vista de sus protagonistas. De igual forma la observación deliberada posibilitó captar el conjunto de actitudes personales y motivacionales, tanto individuales como colectivas, que permitieron calibrar la predisposición a generar un clima favorable a la creación y globalización de conocimientos.

\section{Microcomunidades de conocimiento en el contexto universitario}

Una de las características principales de los grupos de investigación en Venezuela, es que son organizaciones que se originan, desarrollan, perduran y/o desaparecen en otra organización mayor que las arropa: las universidades. Como parte de esa institución, deben respetar normas y valores, así como cumplir funciones que de una u otra manera legitiman su función dentro de la universidad. No obstante, por esas características propias de estas organizaciones, que según Picón (1994), la diferencian de las demás organizaciones sociales, los grupos de investigación establecen sus propios principios de conformación que los diferencian del resto de la organización universitaria. La tesis de este autor sobre el aprendizaje organizacional en las universidades venezolanas se corresponde con los postulados esenciales de la estructura académico-administrativa de la 
Universidad del Zulia y en especial, del Núcleo Agropecuario. En este sentido, Picón (1994), afirma que la Universidad comparte elementos comunes con las demás organizaciones sociales que conforman las sociedades en nuestros días. Como todas ellas, acepta la definición del sistema social abierto, que incorpora recursos de su entorno, procesa esos recursos de una cierta manera, y los devuelve convertidos en un determinado producto, además reincorpora luego nuevos insumos para reenergizarse y mantenerse en el tiempo. Como toda organización formal, está integrada por personas que desempeñan diferentes roles, se rigen por normas establecidas por la organización y comparten un conjunto más o menos uniforme de valores.

Chavoya (2001), infiere que el peso de las disciplinas en la organización del trabajo académico ha hecho que, en muchos estudios sobre la ciencia y los científicos, las instituciones se desvanezcan o, cuando mucho, aparezcan como telón de fondo; sin embargo, no debe soslayarse el estudio de la universidad, no sólo como trasfondo del trabajo de los investigadores, sino como una parte esencial del mismo. Asimismo, la enorme variación de los sistemas universitarios hace necesario destacar las características nacionales y locales de las universidades para entender de qué manera favorecen o limitan los avances disciplinarios y por ende, de sus comunidades académicas.

\section{Aspectos fundamentales de la gestión del conocimiento}

La gestión del conocimiento surge a mediados de la década de 1990, como una alternativa al modelo gerencial basado en la reingeniería, el cual estaba siendo objeto de críticas por parte de los estudiosos de la gerencia, en tanto que la aplicación de esta herramienta organizacional destruyó los bancos de reserva implícitos en la memoria corporativa, con lo cual se suprimía el conocimiento corporativo acumulado a través de la experiencia, mediante la reconstrucción de procesos y estrategias para abordar el nuevo entorno. A diferencia de la reingeniería, la gerencia del conocimiento valora el conocimiento acumulado en la memoria corporativa, asume una vigilancia constante de este conocimiento y estimula la modificación e innovación permanente para responder al entorno cambiante del mercado Koulopoulos y Frappaolo (2000). Su principal representante es Ikujiro Nona$\mathrm{ka}$, quien junto a sus discípulos y colaboradores ha desarrollado el marco teórico para abordar el conocimiento como recurso fundamental en las organizaciones. Sin embargo "...se puede afirmar que la gestión del conocimiento es un área compleja y heterogénea por lo que, actualmente, no se puede afirmar que exista un marco sólido consensuado acerca del conocimiento y su gestión" (Navarro, 2007: 6).

En la gestión de conocimiento empresarial se incorpora el conocimiento de las personas como un activo importante de la empresa, ya que tiene características específicas que dificultan su imitación y su transferencia y por tanto, aquella empresa que gestione de forma eficiente su conocimiento dispondrá de una mayor ventaja competitiva en el mercado. Al respecto, sólo alcanzarán el éxito las empresas que de un modo consistente 
creen nuevo conocimiento, lo difundan por toda la empresa y lo incorporen rápidamente a nuevas tecnologías y productos González et al. (2001). "Además de las prácticas destinadas a acceder a nuevos conocimientos, la gestión del conocimiento incluye métodos para poner en común y utilizar este conocimiento, lo que implica la instauración de sistemas de valores para compartir el conocimiento y de prácticas para la catalogación de los procedimientos" (OCDE y EUROSTAT, 2006). Esta afirmación conduce hacia la noción de conocimiento como concepto fundamental de la gestión de conocimiento dentro de la organización En este trabajo, se adoptó la definición de Von Krogh et al. (2001), de microcomunidades de conocimiento, en tanto que esta resulta compatible con la representación del proceso para la producción de conocimiento, descrito por Picón (1994) sobre los grupos de investigación en Venezuela y por su operatividad a fin de explicar el objeto de este estudio.

Según Von Krogh et al. (2001), el conocimiento tiene ciertas características, tales como: que el conocimiento es una certeza justificada, pues un individuo justifica la veracidad de sus certezas con base a observaciones del mundo, estas observaciones dependen a su vez de un punto de vista personal y experiencia individual peculiar. Los seres humanos justifican permanentemente sus certezas con base a sus experiencias. En el curso de una vida, un individuo adquiere certezas por intermedio de su formación e integración a una familia; educación y capacitación; expectativas sociales; estados emocionales y momentos decisivos; tareas laborales, gustos y preferencias afincados en numerosas experiencias. Cada vez que un individuo confronta un nuevo elemento sensorial, aborda el nuevo estímulo cargado de sus experiencias y certezas sobre el mundo. De acuerdo a esta definición, el conocimiento es una construcción de la realidad más que algo cierto en sentido abstracto o universal. La creación de conocimiento no es simplemente una compilación de datos, sino un proceso propiamente humano que no puede menospreciarse ni reproducirse con facilidad. El conocimiento en sí mismo es mutable y puede adoptar muchos rostros en una organización.

Del mismo modo el conocimiento es tanto "explícito" como "tácito", es decir, ciertos conocimientos son susceptibles de escribirse, formularse en enunciados o representarse en imágenes. El conocimiento explícito es el que se transmite a través de medios tangibles tales como dibujos y especificaciones, con lo cual se vuelve explícito lo que se sabe. Sin embargo, otros tipos de conocimiento se asocian con los sentidos, las habilidades motrices, la percepción individual, experiencias físicas, reglas prácticas generales y la intuición, en este renglón se incluye el conocimiento tácito. Pero aunque la idea del conocimiento tácito es intuitivamente lógica para la mayoría de las personas, a los administradores suele dificultárseles enormemente entenderlo y expresarlo en un nivel práctico. Reconocer el valor del conocimiento tácito e idear cómo usarlo es uno de los más grandes desafíos de una organización creadora de conocimiento, reto este que implica amplias conversaciones y buenas relaciones personales, esto es, facilitación de conocimiento. 
Adicionalmente, la eficaz creación de conocimiento depende de un contexto propicio, es decir, de un espacio común en el que se fomente el establecimiento de relaciones. Sobre la base de la idea japonesa del $\mathrm{Ba}$ (lugar) ese contexto de organización puede ser físico, virtual, mental o las tres cosas al mismo tiempo. El Ba en esencia, es un espacio compartido que sirve de base a la creación de conocimiento. Esta definición de contexto se vincula con los dos puntos anteriores: el conocimiento es dinámico, relacional y se basa en la acción humana; depende de la situación y las personas implicadas, no de una verdad absoluta o de realidades incontrovertibles. Todo conocimiento, en oposición a la información o los datos, depende de su contexto. Podría decirse que el conocimiento está implícito en el $\mathrm{Ba}$, y que para sustentar en su totalidad el proceso de creación de conocimiento es necesario el contexto o espacio de conocimiento adecuado.

Por último, la creación de conocimiento organizacional supone cinco pasos principales que se identifican como factores facilitadores del conocimiento en el Cuadro 1. "En torno a este aspecto, es preciso acotar que la implementación de metodologías apropiadas para la creación deliberada, uso y reutilización de conocimiento organizacional en forma sostenida y permanente, supera la visión de la generación y compartición de conocimiento de manera episódica como respuesta a situaciones emergentes" (Colina, 2007: 209). Por lo que estos factores constituyen metodologías traducidas en prácticas rutinarias organizacionales para la generación y uso efectivo del conocimiento.

\subsection{Las microcomunidades de conocimiento}

Son pequeños grupos de comunidades de conocimiento en el interior de una organización, cuyos miembros comparten lo que saben, además de valores y metas. El éxito de la creación de conocimiento depende de la relación de éstos y otros miembros de las organizaciones, a través de las diferentes etapas del proceso. Las grandes comunidades de conocimiento pueden compartir ciertas prácticas, rutinas y lenguaje; pero el surgimiento de nuevo conocimiento tácito a través de la socialización impone la necesidad de grupos reducidos: de cinco a siete personas. No por ello estas microcomunidades dejan de ser comunidades, con la densa red de relaciones que el término implica Von Krogh et al. (2001).

Más allá de las acciones de actores individuales, la gente trabaja en grupos para crear conocimiento. No obstante, los autores introducen el término microcomunidades de conocimiento como una manera de ampliar el concepto de equipos. Una microcomunidad de conocimiento posee mayor potencial de evolución en el tiempo, porque en teoría hay una mayor cohesión entre sus integrantes, en consecuencia, desarrollará sus propios rituales, lenguaje, prácticas, normas y valores. En cuanto a su conformación, generalmente son creadas desde afuera por un jefe de departamento, gerente de investigación y desarrollo o ingeniero responsable del desarrollo de un producto. En este caso, la tarea de la comunidad, la pertenencia a ella, los beneficios de tal pertenencia y la distinción se basan en iniciativas de la compañía, de 


\section{Cuadro 1 \\ Factores facilitadores del conocimiento}

Factor facilitador

Descripción de la acción

del conocimiento

Inculcar una visión del conocimiento

Conducción de conversaciones

Movilización de activistas del conocimiento

Creación del contexto adecuado

Globalización del conocimiento local
La visión de cualquier organización consta tanto de un componente presente como de un componente futuro. Por visión se entiende no solamente la previsión de un estado futuro, pues, también es necesaria una visión de la situación presente. La visión del conocimiento se relaciona firmemente con una estrategia de avance, en la que se ponen de relieve el desempeño y el éxito futuros de una organización.

Las buenas conversaciones son el origen del conocimiento social en toda organización. La conducción de conversaciones afecta no sólo la compartición de conocimiento tácito, sino todas las demás fases del proceso de creación del conocimiento. En las microcomunidades productivas, las conversaciones pueden liberar las potencialidades creativas de los individuos e incitar la creación de conocimiento más allá de las capacidades de una sola inteligencia (Galvi, 1996, citado por Von Krogh, Ichijo y Nonaka, 2001). A medida que se desenvuelve una conversación interesante, la distinción entre el individuo y el grupo se desvanece.

La facilitación de nuevo conocimiento depende de la energía y compromiso sostenido que una organización invierta en la creación del conocimiento. La participación de activistas del conocimiento es decisiva al menos en cuatro de los pasos de la creación de conocimiento. Los activistas son los responsables de energizar y enlazar los esfuerzos de creación de conocimiento en toda la organización. Pese a que es poco frecuente que intervengan directamente en la compartición de conocimiento tácito en microcomunidades y grupos aún más pequeños, los activistas del conocimiento contribuyen a establecer el contexto facilitador adecuado, el espacio y las relaciones esenciales para la liberación de conocimiento tácito.

Este factor tiene que ver con las estructuras de la organización que fomenta la existencia de relaciones sólidas y una colaboración eficaz. Se requiere de estructuras flexibles es decir, los organigramas tradicionales caracterizados por la rigidez de sus jerarquías y la integración vertical, ya no son aptos para la coordinación de actividades de negocios en un mundo en el que los linderos se han desvanecido, las relaciones son cada vez más complejas y el ambiente competitivo se halla en constante estado de flujo. La clave es estructurar una organización en la que la creación de conocimiento proceda con mayor eficacia y eficiencia, lo cual supone desmantelar tantas barreras individuales y organizacionales como sea posible.

Este factor ejerce un impacto positivo en la formación de microcomunidades, especialmente en sitios de destino del conocimiento creado. Sin embargo, la globalización del conocimiento local influye directamente en el modo de compartir conocimiento tácito en el interior de las microcomunidades, la creación de conceptos, la justificación de conceptos y la elaboración de prototipos, ya que en general éstos son procesos autónomos. La principal razón de ser de este facilitador es la difusión del conocimiento en una organización.

Fuente: Elaboración propia con base en Von Krogh, Ichijo y Nonaka (2001). 
manera que la comunidad opera en forma de equipo. Sin embargo, el surgimiento de microcomunidades de conocimiento también puede ser resultado de la autoorganización. En un principio, los miembros podrían componer una organización informal y compartir un lugar común en el tiempo y en el espacio, física o virtualmente.

En esta etapa, el conocimiento es individual; pero conforme se intensifica la interacción de los miembros, estos establecen relaciones recíprocas, hablan de temas generales, quizá programan varias reuniones futuras; esto es, forman un grupo más coherente. Tras la identificación de intereses comunes, un grupo fusionado puede convertirse en "grupo comprometido". En este caso, el grupo organiza los derechos y deberes de sus miembros de acuerdo con un juramento o estatuto. El conocimiento explícito del grupo se complementa con el conocimiento del juramento, así como con el conocimiento tácito adicional del apasionamiento, del compromiso y las emociones positivas que experimentan los miembros. Lo que los citados autores denominan microcomunidad, es la puesta en acción de un grupo comprometido, el inicio de éste en la creación de conocimiento. Una microcomunidad fomenta, asimismo, la coherencia interna mediante el realce de los beneficios de pertenecer a ella tales como: placer, identificación de intereses, satisfacción de necesidades y retribuciones futuras. Una vez disuelta y desmantelada una microcomunidad, gran parte de su conocimiento tácito se pierde, especialmente el tipo de conocimiento que implica relaciones entre los miembros de la comunidad.

\subsection{Características de las distintas modalidades de organización del proceso de generación de conocimiento en el Núcleo Agropecuario de la Universidad del Zulia}

En el Cuadro 2 se ofrece una caracterización general, la cual es recogida en una tipología de formas de organización del proceso de generación y globalización de conocimiento, como resultado del estudio de los diferentes grupos de investigación adscritos al núcleo agropecuario de LUZ, antes citados. Dicho estudio permitió identificar distintas formas de organización y tratamiento en torno a la creación y uso adecuado del conocimiento generado por dichos grupos, lo que implica a su vez la distinción de diferentes de actitudes asumidas, aptitudes desarrolladas y fortalecidas, prácticas y métodos organizacionales entre otros aspectos, de orden intangible inherentes a los grupos. Conllevando en consecuencia a la distinción de los tipos I, II y III.

Los grupos estudiados y especialmente los clasificados como microcomunidades de conocimiento, comparten todos o casi todos los elementos característicos de las microcomunidades de conocimiento definidas por Von Krogh et al. (2001) y Picón (1994); sin embargo, en función de las particularidades propias de la institución universitaria, como organización generadora de conocimiento, se puede afirmar que se está en presencia de "Microcomunidades Académicas de Conocimiento". Dicha afirmación se fundamenta en los parámetros que a continuación se señalan en el Cuadro 3 , los cuales resultan de gran utilidad a fin de 


\section{Cuadro 2 \\ Aproximación a una tipología de formas de organización del proceso de generación de conocimiento en el Núcleo Agropecuario de LUZ}

Tipo I: Microcomunidades

Académicas

de Conocimiento
Tipo II: Equipos de

Investigación en fase de

transición hacia

Microcomunidades

Académicas de Conocimiento

Conformadas por un número de integrantes de carácter permanente que oscila entre los 5 y los 7 miembros, pero en su dinámica conforman una red de interrelaciones que incorpora a una gran cantidad de personas e instituciones.

Sus integrantes comparten la misma visión y esa visión es la que rige sus acciones.

Los problemas son abordados bajo el enfoque sistémico.

El liderazgo es compartido entre un equipo coordinador.

Sus líderes son verdaderos activistas del conocimiento.

Establecen una rutina de conducción de conversaciones marcada por la organización, la planificación y la periodicidad, sin limitar la espontaneidad.

Son agrupaciones con un número de integrantes de carácter permanente que oscila entre los 5 y los 7 integrantes, pero pueden incorporar a una gran cantidad de personas e instituciones.

Tienen definida su misión y su visión y las mismas son compartidas por todo el grupo.

No asumen un enfoque sistémico en la forma de abordar los problemas de investigación como tampoco en la organización del equipo como tal.

Son grupos transdisciplinarios. No son grupos transdisciplinarios.

El liderazgo es centralizado.

Sus líderes son activistas del conocimiento.

Establecen una rutina de conducción de conversaciones marcada por la organización, la planificación y la periodicidad, sin limitar la espontaneidad.
Tipo III: Grupos

de Investigación
Son grupos altamente productivos, enmarcados en una rutina de trabajo que se apoya en formas tradicionales de la organización de la investigación y pueden sobrepasar a los 7 miembros.

Algunos de sus miembros comparten una misma visión y otros no, derivado de la diversidad de valores en sus integrantes.

No son sistémicos en sus enfoques y usualmente no se integran en redes por lo que predomina el trabajo aislado e individual.

No se visualiza la transdisciplinariedad en su modelo de investigación ni en la estructura de su organización como grupo.

En términos generales tanto el liderazgo como las decisiones están centralizadas en una sola persona.

Sus líderes no siempre son activistas del conocimiento.

No establecen rutinas para la conducción de conversaciones como metodología organizacional de creación y compartición de conocimientos. 


\section{Cuadro 2 (Continuación)}

Tipo I: Microcomunidades

Académicas

de Conocimiento
Tipo II: Equipos de

Investigación en fase de

transición hacia

Microcomunidades

Académicas de Conocimiento

Establecen una planificación

de actividades anuales.

Plantean objetivos

estratégicos en función de la visión.

Exhiben elevada producción académica con evidencias de impacto en el medio intervenido.

Conciben la integración de las tres funciones básicas de la universidad (investigación-docencia y extensión) como una trilogía indisoluble e indispensable para el alcance de sus metas.

Entre sus integrantes se evidencia armonía y una profunda relación de amistad que muchas veces traspasa las fronteras del recinto universitario.

Son grupos altamente innovadores.

Conocen a sus clientes y usuarios y saben hacia donde quieren ir y cómo hacerlo. Así mismo estos se tornan una fuente de información relevante que luego es convertido en conocimiento con significado.
Plantean objetivos estratégicos en función de la visión.

Evidencian una elevada producción académica de impacto en el medio intervenido.

Enfocan los problemas integrando las tres funciones básicas de la universidad.

Establecen lazos laborales y personales muy estrechos y de armonía.

Están ganados al cambio y a la innovación.

Sus usuarios y clientes ocupan un lugar importante en las agendas investigativas, por el caudal de conocimientos que estos aportan para presentes y futuras investigaciones.
Tipo III: Grupos

de Investigación
La planificación de sus actividades básicamente está limitada a la rendición de cuentas ante el CONDES y el FONACYT.

Los objetivos a menudo pueden ser objeto de divergencias en virtud de que no siempre se comparte la visión.

Su productividad académica aunque es abundante no siempre impacta la realidad estudiada.

No siempre se alcanza a integrar las tres funciones básicas de la universidad.
En ciertos casos se observan divergencias y rivalidades entre algunos miembros, pues se busca sobresalir individualmente y no como equipo.

Son grupos poco ganados al cambio y a la innovación.

Establecen relaciones con entes externos al grupo únicamente como fuente alternativa para la obtención de recursos de diversa índole y sobre esta base conciben y desarrollan la extensión de los resultados de investigación.

Fuente: Elaboración propia. 


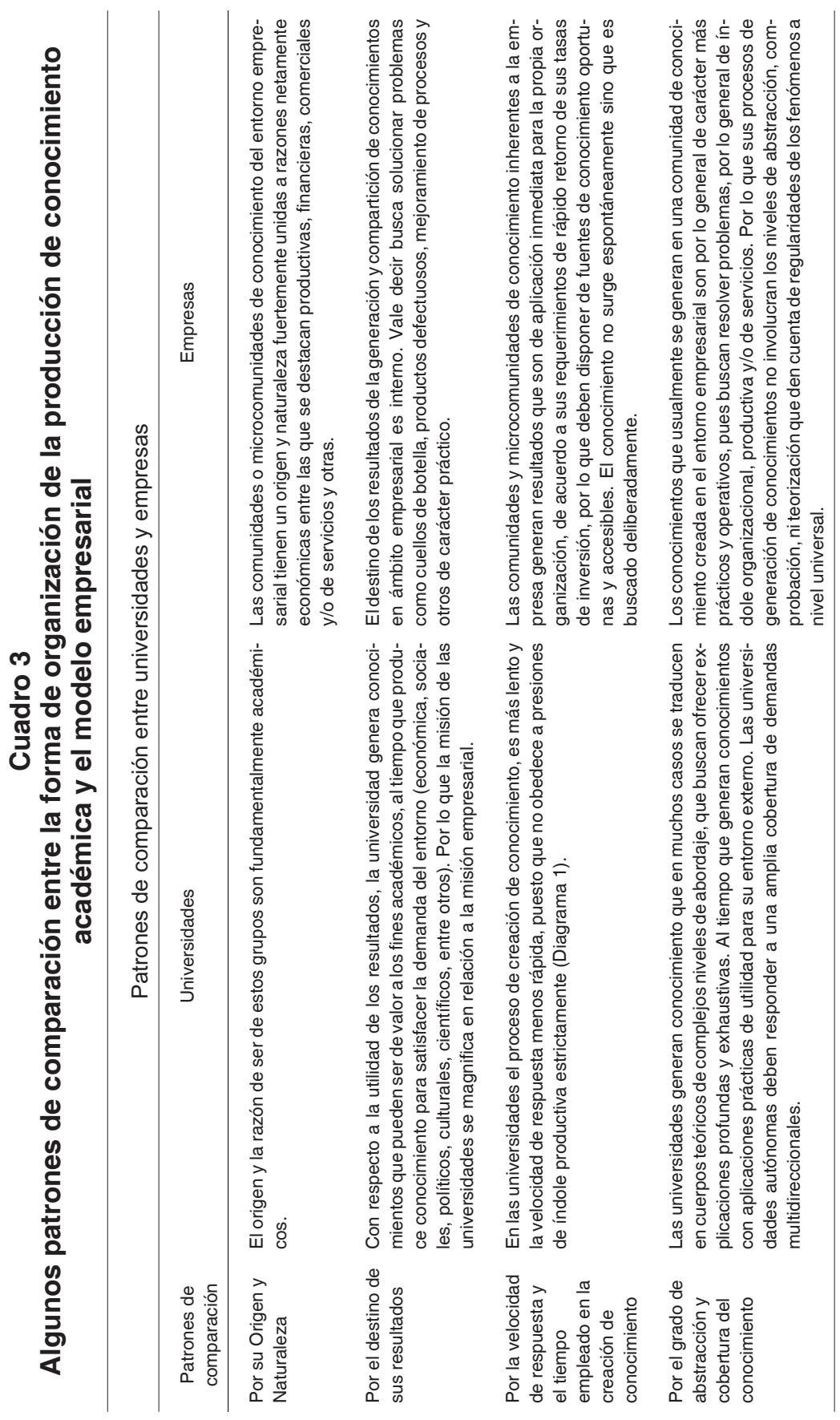




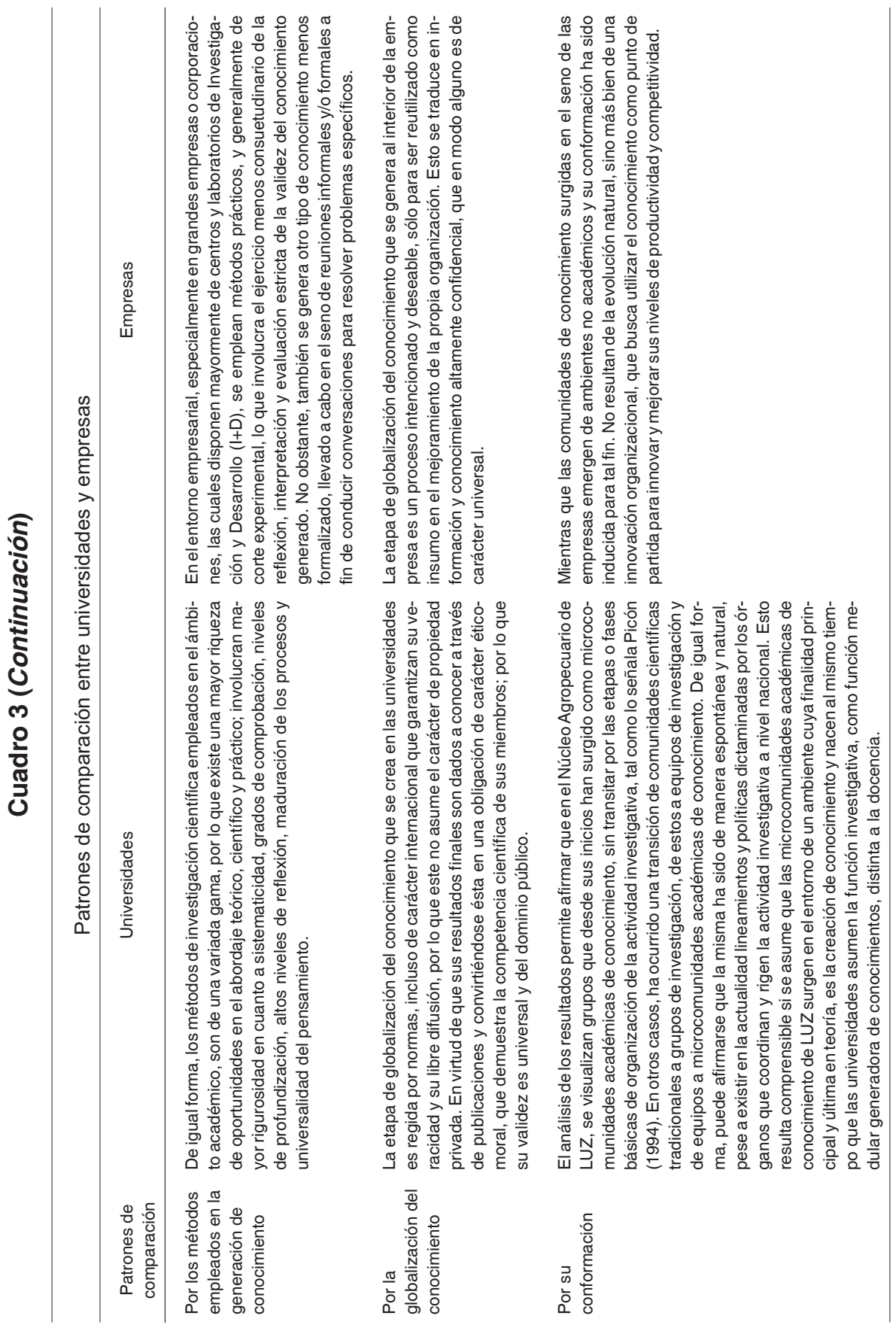




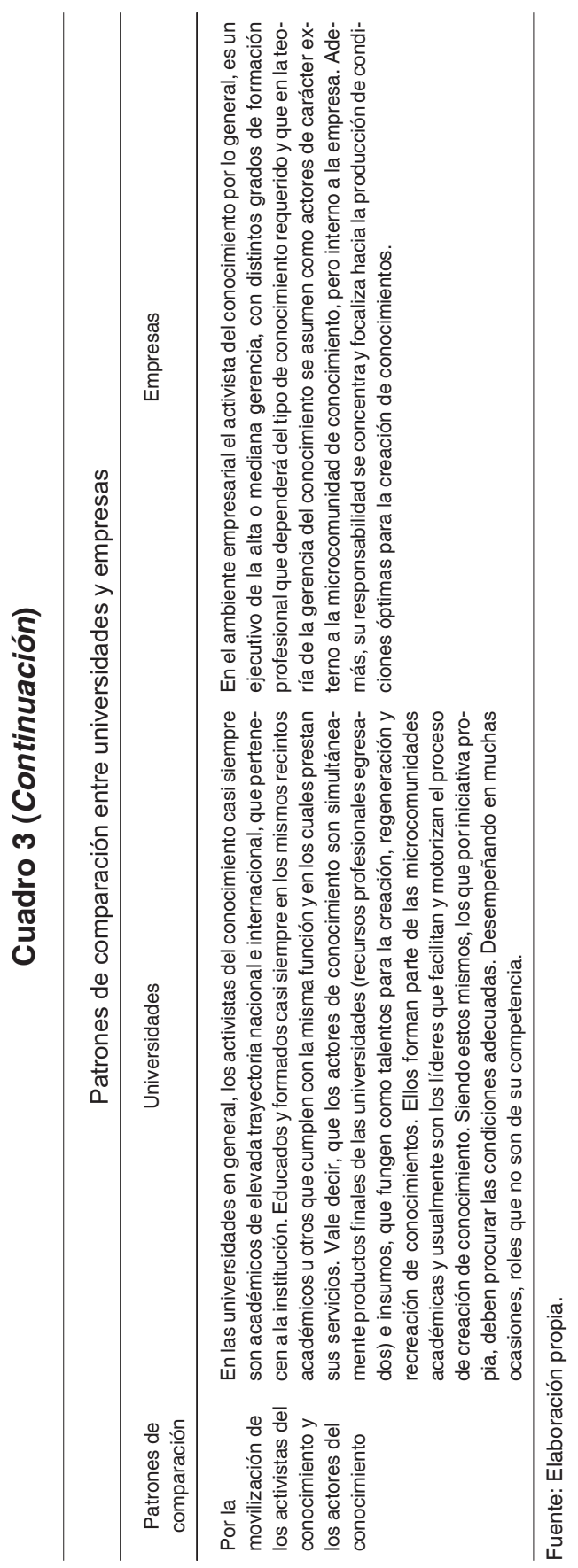




\section{Diagrama 1}

\section{Ciclo de creación de conocimiento en una microcomunidad académica} de conocimiento de una universidad autonóma

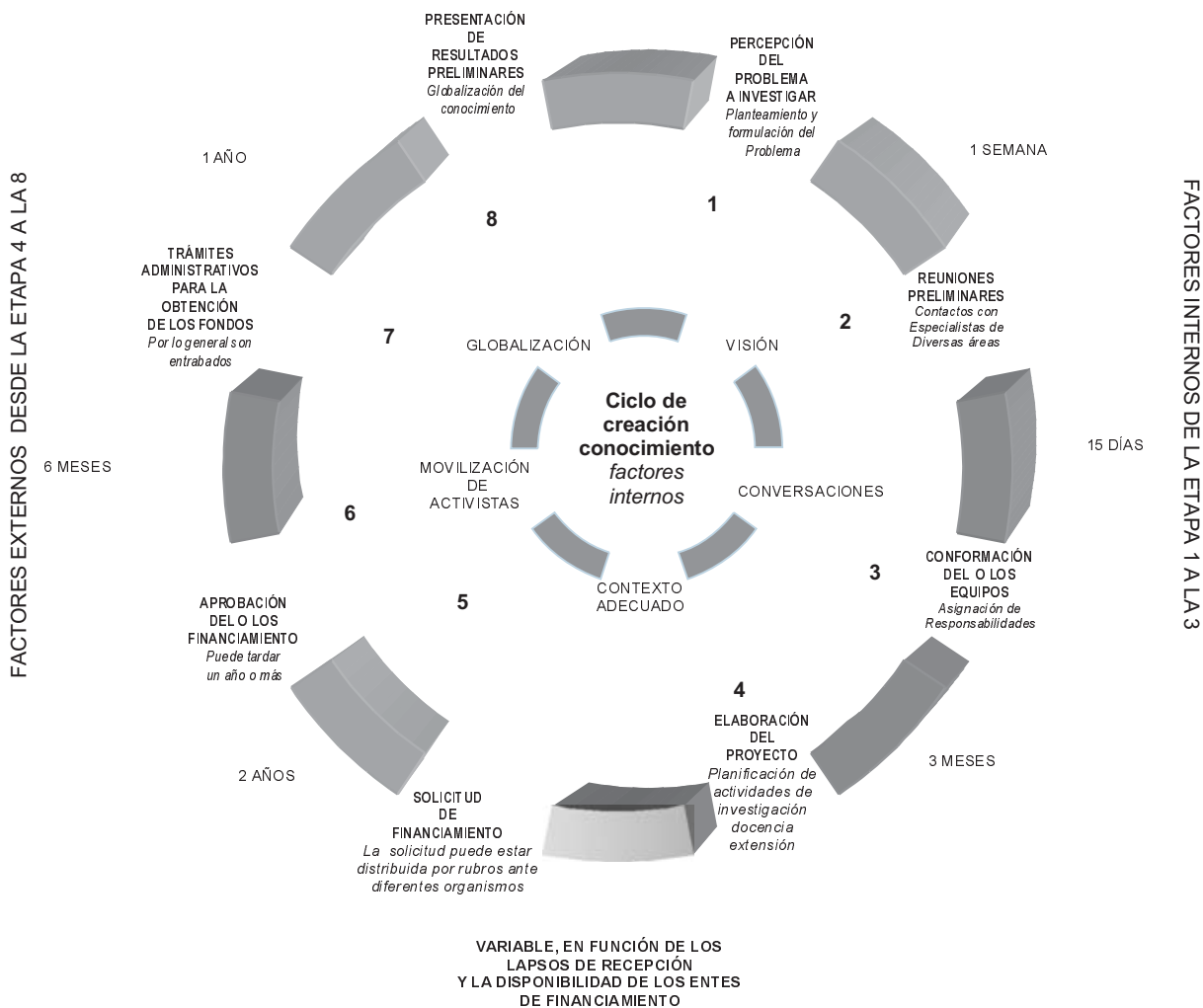

Fuente: Elaboración propia (2007).

establecer algunos patrones de comparación con respecto a la particular forma de organización de la producción de conocimiento académica en relación al modelo empresarial aportado por Von Krogh et al. (2001).

\subsection{Características académicas}

\section{de los grupos estudiados}

Antigüedad en las labores académicas:

De los resultados obtenidos en este aspecto, llama la atención que el
$50 \%$ de los integrantes de los grupos de investigación, poseen una antigüedad en labores académicas entre los 5 y 15 años (Gráfico 1) Este dato resulta interesante en tanto que revela que los grupos están compuestos, fundamentalmente, por gente joven. Ante la crisis de recursos humanos que vive actualmente la universidad, debido a la grave situación financiera que ha impuesto restricciones para el ingreso de personal académico, resultan alentadoras las cifras de gente joven dedicada a la investigación. Otro aspecto 
importante en este análisis es la necesidad de relacionar este resultado con la política de ingreso a través del Programa de Becarios Académicos de LUZ y su homólogo, el Programa de Investigadores Noveles del FONACYT, mediante los cuales ingresa personal académico joven -menor de 35 años-, condicionado al cumplimiento de un plan de formación que incluye, entre otras actividades el desarrollo de un proyecto de investigación y la realización de estudios de maestría. Con esta estrategia, se instruye al joven aspirante acerca de lo que deben ser sus obligaciones, una vez ingresado como personal ordinario, a la vez de fomentar el interés por la investigación.

Acreditación en programas de reconocimiento por la labor académica:

El $42,86 \%$ de los integrantes de los grupos de investigación está acreditado por el Sistema de Promoción del Investigador, a través del Programa de Promoción del Investigador (PPI) (Gráfico 2). Este dato evidencia una gran producción científica de los grupos que luego es reconocida individualmente a través de este programa. El elevado número de profesores adscritos al PPI llama la atención en virtud de que el 47,62 de los inte-

\section{Gráfico 1 \\ Años como integrante del grupo con base a un promedio de todos los grupos estudiados}

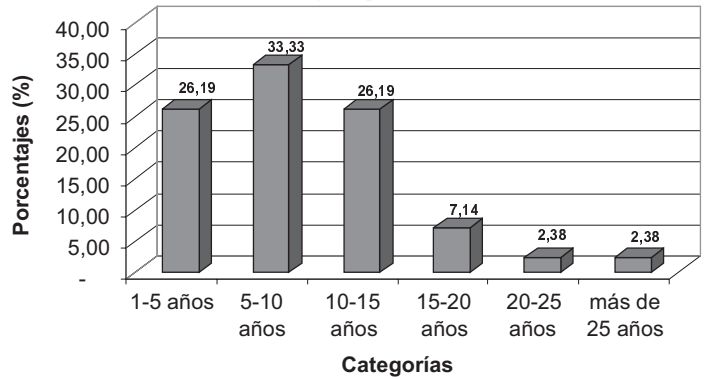

Fuente: Elaboración propia.
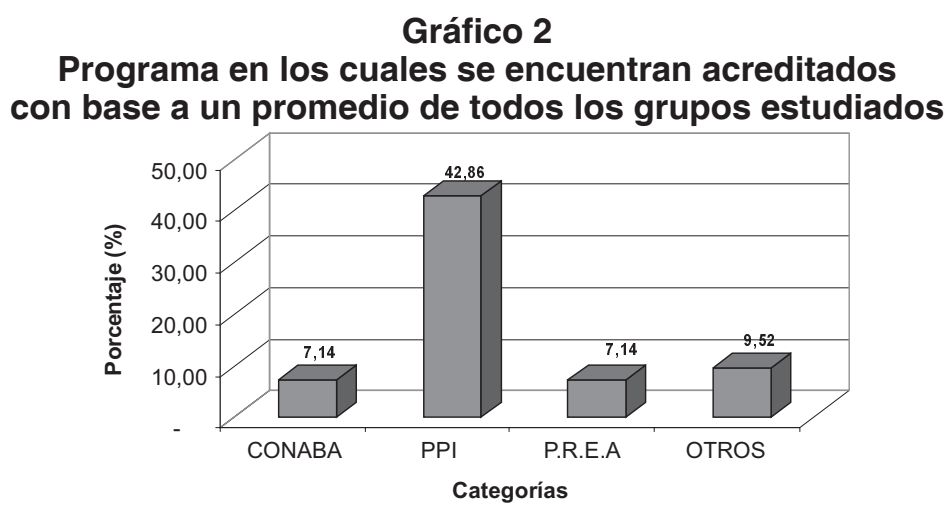

Fuente: Elaboración propia. 
grantes posee el nivel de maestría y tomando en cuenta el nivel de exigencia de este programa, en cuanto al número de publicaciones para el primer ingreso (10 publicaciones para el nivel 1 y 1 para e nivel candidato) así como la obligatoriedad de al menos una publicación durante el período de dos años, se puede concluir que la producción científica de estos investigadores es significativamente alta.

Número de publicaciones científicas y de extensión:

La Tabla 1 muestra el número de publicaciones científicas y de extensión

\section{Tabla 1}

\section{Producción total de publicaciones científicas y de extensión generadas por los integrantes de los grupos/equipos y microcomunidades académicas de conocimiento estudiados en toda su trayectoria}

\begin{tabular}{|c|c|c|c|}
\hline $\begin{array}{l}\text { Grupo/equipo/micro- } \\
\text { comunidad académica } \\
\text { de conocimiento } \\
\text { adscritos al Núcleo } \\
\text { Agropecuario de la } \\
\text { Universidad del Zulia }\end{array}$ & $\begin{array}{l}\mathrm{N}^{\circ} \text { de publicaciones } \\
\text { científicas total en la } \\
\text { trayectoria académica }\end{array}$ & $\begin{array}{c}\mathrm{N}^{\circ} \text { de publicaciones de } \\
\text { extensión total en la } \\
\text { trayectoria académica }\end{array}$ & $\begin{array}{l}\text { Total publicaciones } \\
\text { científicas y de } \\
\text { extensión en la } \\
\text { trayectoria académica } \\
\text { de cada grupo }\end{array}$ \\
\hline Frutales & 106 & 21 & 127 \\
\hline $\begin{array}{l}\text { Grupo de Diagnóstico } \\
\text { de la Unidad de } \\
\text { Investigación Clínica }\end{array}$ & 71 & 61 & 132 \\
\hline $\begin{array}{l}\text { Investigación en } \\
\text { Reproducción Animal } \\
\text { del Zulia (GIRAZ) }\end{array}$ & 103 & 66 & 169 \\
\hline $\begin{array}{l}\text { Museo de Artrópodos } \\
\text { (MA) }\end{array}$ & 51 & 6 & 57 \\
\hline $\begin{array}{l}\text { Programa de } \\
\text { Investigación en } \\
\text { Comunidades Agrícolas } \\
\text { (PICA) }\end{array}$ & 30 & 13 & 43 \\
\hline $\begin{array}{l}\text { Sistemas de } \\
\text { Información en } \\
\text { Ganadería de Doble } \\
\text { Propósito }\end{array}$ & 52 & 28 & 80 \\
\hline $\begin{array}{l}\text { Tecnología de } \\
\text { Alimentos }\end{array}$ & 79 & 9 & 88 \\
\hline $\begin{array}{l}\text { Ciencia y Tecnología de } \\
\text { la Carne }\end{array}$ & 158 & 24 & 182 \\
\hline $\begin{array}{l}\text { Total Publicaciones en } \\
\text { la trayectoria académica }\end{array}$ & 650 & 228 & 878 \\
\hline
\end{tabular}

Fuente: Elaboración propia. 
total en términos absolutos, que a lo largo de su carrera han generado los integrantes de cada uno de los grupos, equipos o microcomunidades académicas de conocimiento estudiados. En este sentido, se corrobora una elevada producción científica, si tomamos en cuenta que según las normas vigentes del Consejo de Desarrollo Científico y Humanístico (CONDES) de la Universidad del Zulia, así como las del Programa de Promoción del Investigador (PPI), para ser un investigador activo basta con tener por lo menos una (1) publicación por cada dos años. Aunque no era objeto de este estudio evaluar la producción científica de los grupos estudiados, se consideró pertinente presentar el conjunto de su producción académica como un indicador relevante a fin de complementar la información relacionada con las características de los mismos. Por otra parte, es importante señalar que el conjunto de las publicaciones de los grupos estudiados, representan también parte de la productividad académica de la Facultad respectiva en particular y de la Universidad del Zulia en general.

Otro aspecto importante a destacar es que el número de publicaciones no está directamente relacionado con el tipo de grupo en el cual se ubican, esto quiere decir, que aún los grupos tradicionales poseen una alta producción científica y de extensión. Esta elevada producción tiene su razón de ser en la convicción de los investigadores de que los resultados de toda investigación científica deben ser globalizados y confrontados con sus pares a través de las publicaciones. De hecho, en el ambiente de estos grupos se maneja la tesis de que "investi- gación que no se publica, no se conoce, por lo tanto no existe".

Por otra parte, también pudiera ser el resultado de la política para el incremento de la producción científica de LUZ, implantada por el CONDES en el año 1993, mediante la cual por cada millón de bolívares aprobado para el proyecto $o$ programa de investigación, el investigador responsable debería reportar en el informe final al menos una (1) publicación al año.

\section{Factores potencializadores del proceso de creación de conocimiento en el Núcleo Agropecuario de LUZ}

La característica principal de estos grupos es la tendencia a funcionar con una baja formalización de y con base en relaciones predominantemente horizontales. Con excepción del GIRARZ, que a raíz de su conformación formalizó su sistema de organización, los grupos de investigación no poseen una estructura organizacional formalmente establecida. Usualmente existen coordinaciones por áreas, liderados por las personas más influyentes en cada una de ellas. La autoridad se establece en función del conocimiento y en lo esencial se rigen por un conjunto de valores entre los cuales sobresalen: la toma de decisiones en conjunto, el reconocimiento del sentido de compromiso de los integrantes y su capacidad de respuesta ante las tareas asignadas, el compartir la información y los problemas; al igual que el respeto a la autoridad basada en el conocimiento. Los factores potencializadores pueden visualizarse de una forma global en el Diagra- 
ma 2 y son analizados en detalle en los puntos que siguen.

\subsection{Inculcar una visión del conocimiento}

Partiendo del punto de vista de que la visión no es un valor, pero sí es reflejo del sentido de compromiso del grupo con la misión en este caso de la universidad, en el conjunto de valores que sostienen los grupos estudiados se consideró y se destacó como punto especial este aspecto, en virtud de la importancia que los grupos manifestaron hacia el sostenimiento de la visión del grupo, sobre todo las microcomunidades académicas de conocimiento. En este sentido, resulta interesante el hecho de que aunque la mayoría de los grupos no tengan definida formalmente su visión, el lineamiento acerca de lo que ellos consideran cual es la visión del grupo, es compartido por todos los miembros y en función de ello se orientan las estrategias. Esta visión facilita la cohesión del grupo y la auto exclusión de los que no la comparten. Un aspecto ligado al anterior es que todos los grupos estudiados realizan actividades simultáneas de investigación, docencia y extensión, no obstante es la actividad de extensión la que actualmente genera mayores grados de satisfacción, desde el punto de vista de su impacto social, lo cual viene a representar un componente indispensable de su visión. El ámbito de aplicación de sus trabajos de extensión, tal como las unidades productivas, entre otras, se convierten en sus laboratorios y aulas de clase privilegiada; y en el proceso comunicacional que se establece, se produce una retroalimentación de conocimientos

\section{Diagrama 2}

Factores potencializadores de la creación de conocimiento en las microcomunidades académicas de conocimiento del Núcleo Agropecuario de la Universidad del Zulia

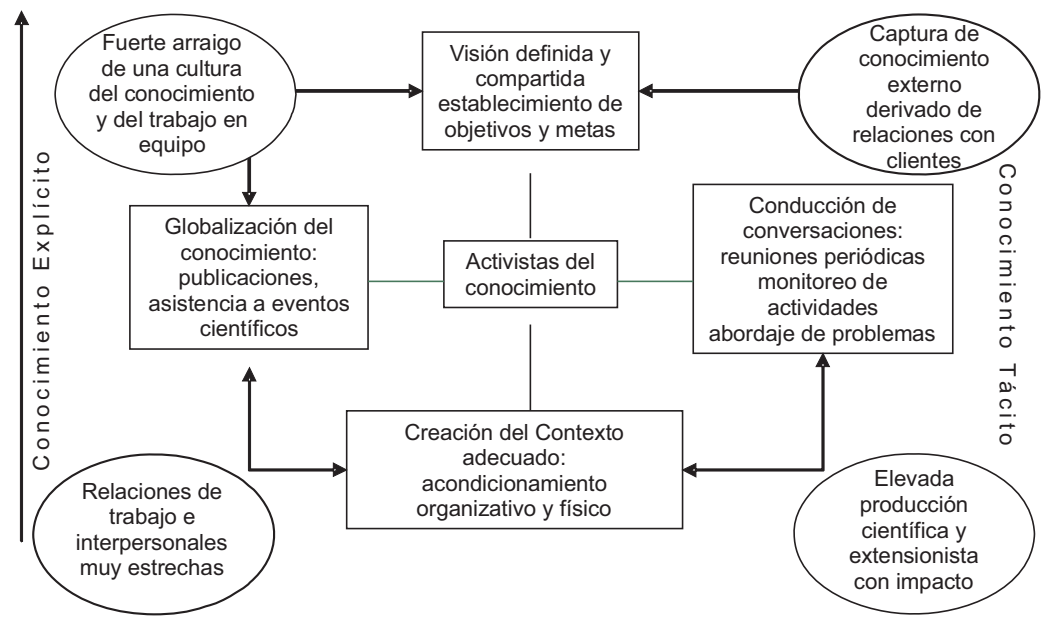

Fuente: Elaboración propia. 
entre las comunidades y los investigadores, que a su vez son fuente para fortalecer sus investigaciones e impulsar otros estudios y en consecuencia la generación de otros conocimientos.

\subsection{Conducción de conversaciones}

En el campo de la investigación, la conducción de conversaciones se realiza, básicamente

a través de reuniones periódicas o de jornadas de trabajo denominadas seminarios. En dichas reuniones o seminarios, se planifican las actividades del grupo, se delibera sobre el desarrollo de las mismas, se someten a evaluación y se aplican los correctivos necesarios. Al tiempo que las reuniones sirven para resolver conflictos entre el grupo, tratar problemas individuales, establecer estrategias de ayuda y estrechar los nexos entre los integrantes. A menudo, el trato entre los integrantes de un grupo de investigación es familiar, su contacto va más allá de la rutina de trabajo y con frecuencia se extiende al campo familiar. En las conversaciones que se establecen en dichas reuniones se confirma y reconfirma la pericia de los integrantes sobre su línea de trabajo, lo que permite la eficaz resolución de problemas.

\subsection{Movilización de activistas del conocimiento}

En el caso de los grupos estudiados, el coordinador es el que ejerce el mayor peso en la conducción de los procesos para el desarrollo de las actividades. Esto implica el desarrollo de capacidades especiales para incentivar la crea- ción de conocimientos dentro del grupo y en la Facultad, así como para mantener una intensa comunicación con todos los niveles, tanto de la Facultad como de otros organismos para el desarrollo de la investigación en LUZ y en el país. Usualmente el líder o coordinador del grupo, es quien propone la creación de los programas de investigación que dan origen al grupo, o en su defecto por el fundador del mismo. También es el líder o coordinador quien, originalmente, establece la visión y quien instaura los nexos con investigadores de otras Facultades e instituciones. Adicionalmente, es quien orienta la búsqueda de financiamiento para el desarrollo de los proyectos. Esta situación no deja de ser problemática, toda vez que al asumir el rol de activistas del conocimiento y de académicos simultáneamente, se tornan personas con una carga de actividades en muchos casos insostenibles, todo ello aunado a una rutina excesivamente acelerada que algunas veces desencadenan en crisis dentro del grupo. No obstante, estos activistas están dotados de características personales y profesionales especiales, que les permite imprimir una buena dosis de entusiasmo como para superar las crisis y aún así obtener excelentes resultados.

\subsection{Globalización del conocimiento local}

En términos generales los grupos de investigación estudiados generan conocimiento globalizable, por cuanto en la creación de conocimiento científico priva como principio, el carácter universal del mismo. Aun cuando el conocimiento producido tenga como punto de partida un 
problema local, los resultados deben permitir generalizaciones que, haciendo los señalamientos particulares, puedan ser aplicados en otros contextos. Por otro lado, cuando se toman modelos creados en otros contextos geográficos para producir conocimiento, usualmente se recrea dicho conocimiento adaptándolo a las circunstancias locales. Para los integrantes de grupos de investigación es de vital importancia la publicación de resultados en revistas científicas, toda vez que de éstas dependen las calificaciones internas y externas a su producción, así como los reconocimientos individuales por la labor desempeñada.

\section{Cultura organizacional}

Según (Granell et al., 2000: 5-6) “El concepto de cultura organizacional se refiere al conjunto de creencias, valores, supuestos y conductas compartidos y transmitidos en una organización, que son adquiridos a lo largo del tiempo y que han resultado exitosos para el logro de sus objetivos". Además según estos autores la cultura organizacional varía de país en país, por lo que el contexto nacional determina en buena medida las características de esta.

\subsection{Valores}

Resaltan los relacionados con el compañerismo, la responsabilidad y la honestidad. El compañerismo se manifiesta a través de la ayuda mutua y la solidaridad, así por ejemplo, cuando uno de los miembros del grupo se ve impedido para desarrollar una actividad asignada, la misma es asumida por otro de los miembros. El sentido de responsabilidad resulta trascendental por la elevada importancia que le otorgan todos los grupos en general. Las actividades se planifican asignando responsabilidades en función de méritos y las capacidades académicas. El no cumplimiento del deber acarrea problemas al grupo en general, por cuanto de ello depende su capacidad de respuesta ante los organismos que financian las actividades, así como sus relaciones con las instituciones del entorno con las cuales comparten intereses comunes. Finalmente, en el mundo académico ser honesto gira en torno a dos principios: por un lado, evitar el uso indebido de los recursos que se obtienen para el desarrollo de sus distintas actividades y por el otro, no apropiarse de conocimiento ajeno. En líneas generales, estos valores fortalecen la continuidad o persistencia de las acciones del grupo, porque favorecen su cohesión interna, la motivación de sus miembros, la lealtad hacia el equipo, el compromiso con las tareas y la capacidad para enfrentar y resolver situaciones adversas.

\subsection{Motivación}

La recolección de los datos para el análisis de la motivación se realizó a través de dos fuentes: las entrevistas a los coordinadores o líderes de los grupos y en segundo lugar mediante el cuestionario aplicado a los investigadores incluidos en la muestra. Así, se obtuvo que entre el $69 \%$ y el $52 \%$ de los investigadores que respondieron el cuestionario, manifestaron que los factores que más influenciaron su decisión de pertenecer al grupo de investigación están, según el orden de 
preferencia: su vocación como investigador, la necesidad de compartir sus conocimientos con un grupo, el nivel de productividad del grupo y su identificación con la visión del grupo.

\subsection{Normas}

Las respuestas que se obtuvieron de los informantes claves en torno a las normas, reflejan una variedad de criterios para regular el comportamiento de los miembros de los grupos de investigación. Estos resultados concuerdan con los de Picón (1994) y Chavoya (2001) en el sentido de que en ninguno de los grupos existen normas formalmente establecidas, sin embargo existe un acuerdo tácito entre todos sus miembros para el cumplimiento de las mismas. Entre las normas más comunes se destacan: la realización de reuniones periódicas destinadas a la discusión de los asuntos generales relacionados con la planificación, la organización y la evaluación del trabajo. Las mismas constituyen la rutina central para la interacción y el espacio fundamental para la planificación de todas las actividades y la toma de decisiones sobre la marcha general del grupo. Otra de las normas establecidas, está relacionada con el uso de los equipos. Es casi una norma general, hacer turnos para el uso de los equipos y realizar mantenimiento periódico. Esta normativa tiene su razón de ser en el elevado costo de los equipos y las dificultades para acceder a financiamiento para su adquisición. Del análisis del aspecto normativo se desprende que el alto nivel de valoración hacia las normas y su cabal cumplimiento en beneficio de los intereses colectivos y no individuales, se torna un factor cohesionador, que denota homogeneidad en los criterios y que garantiza el éxito de los grupos señalados y definidos como microcomunidades académicas de conocimiento.

\section{Conclusiones}

El conocimiento que se dispone acerca de la ciencia, los científicos y las comunidades científicas resulta poco exhaustivo y directo como antecedentes del tema de las microcomunidades académicas de conocimiento. Tal vacío teórico quizá se deba, entre otras razones, a que la necesaria y a la vez distorsionada relación entre las universidades venezolanas en general y la del Zulia en particular, y el sector externo, no ha producido estímulos suficientes para hacer del conocimiento generado en la academia un objeto de estudio relevante, ni un recurso útilmente atractivo que amerite ser gestionado de manera eficaz y eficiente. Por lo que la organización y reorganización del trabajo intelectual, al interior de las universidades, en general ha sido una tarea intencionalmente poco atendida y sus resultados no siempre han sido satisfactorios, especialmente para posibles demandantes externos.

Los grupos considerados como microcomunidades académicas de conocimiento coinciden con la definición de Von Krogh et al. (2001) en los siguientes aspectos: la pertenencia a ellas se basa en la posesión de experiencias en un área específica del conocimiento (comunidades rurales, tecnología de alimentos, sistemas de información, tecnología de la carne), interés en el desarrollo de ese conocimiento, disposición a compartir este 
conocimiento en la comunidad, y la existencia de lazos personales y valores comunes de justicia, honestidad, responsabilidad, comunicación, confianza y apoyo mutuo, siendo estos los aspectos intangibles que se constituyen en factores condicionantes potencializadores; y que hacen las veces de lazos invisibles que dan soporte a la conformación de estos grupos como microcomunidades académicas de conocimiento, los cuales han demostrado un alto desempeño y rendimiento académico de elevada pertinencia social.

Por otro lado es preciso destacar que estos grupos no actúan como islas de conocimiento, sino que establecen estrechas vinculaciones con otras comunidades por relaciones de intercambio o tareas comunes. Carecen de fronteras explícitas o decálogos que determinen acerca de quienes pueden ser sus miembros, constituyendo sólo el mutuo acuerdo, la compartición de una visión del conocimiento dinámica y la competencia académica los criterios de pertenencia a ellas. Finalmente, se tiene la convicción de que estos grupos constituyen dentro del marco universitario organizaciones con características muy especiales, que los distinguen de otros grupos de investigación pues el elevado impacto de sus investigaciones en la realidad, es un asunto que puede corroborarse accediendo a los registros que estas organizaciones almacenan con especial cuidado y que representan el verdadero valor que puede ostentar cualquier recinto académico.

\section{Referencias Bibliográficas}

Colina A, Belinda E. (2007). Modelos de desarrollo de dinámicas de innovación en
Petróleos de Venezuela, S.A. (PDVSA). Problemas del Desarrollo. Revista Latinoamericana de Economía. Vol. 38-148. Universidad Autónoma de México UNAM. México - Distrito Federal.

Colina A, Belinda E. (2007). Inédito. Gerencia de Conocimiento, Aprendizaje y Cultura del Conocimiento. Anteproyecto de tesis doctoral titulada: "Capacidades Societales de Innovación en Empresas de Producción Social (EPS) en Venezuela". Facultad de Ciencias Económicas y Sociales de la Universidad del Zulia.

Chavoya Peña, María Luisa (2001). Organización del Trabajo y Culturas Académicas. Estudio de dos grupos de investigadores de la Universidad de Guadalajara. Revista Mexicana de Investigación Educativa. Vol. 6, № 11, México, Enero-abril.

González, Nuria; Nieto, Mariano; Muñoz, Felisa (2001). La Gestión del Conocimiento como Base de la Innovación Tecnológica: el Estudio de un Caso". Memorias del X Seminario Latinoamericano de Gestión Tecnológica. Brasil.

Granell, Elena; Garaway, David y Malpica, Claudia (2000). Éxito gerencial y cultura. Retos y oportunidades en Venezuela. Ediciones IESA. 2da. reimpresión. Caracas - Venezuela.

Granell, Elena (2000). “¿Cómo crear una Cultura para Gerenciar el Conocimiento?”. Gerencia del Conocimiento. Potenciando el capital intelectual para crear valor. Fondo Editorial del Centro Internacional de Educación y Desarrollo (FONCIED) PDVSA. Venezuela.

Koulopoulos, Thomas; Frappaolo, Carl (2000). Lo Fundamental y lo más Efectivo Acerca de la Gerencia del Conocimiento. SMART. Mc Graw Hill. Colombia. 
Navarro de G., Ketty; Romero de G, Eunice; Bauza, Roberto y Granadillo, Víctor (2006). Estudio sobre la gestión tecnológica y del conocimiento en una organización creadora de conocimiento. Revista Venezolana de Gerencia. Año 11. No. 34. Abril-Junio.

Navarro de G., Ketty (2007). Inédito. Anteproyecto de tesis doctoral, titulada: Estrategias de Gestión del Conocimiento de las Empresas Familiares de la región Castilla La Mancha, España. Universidad de Castilla La Mancha, España. Facultad de ciencias económicas y empresariales. Ciudad Real-España.

Organización para la Cooperación y el Desarrollo Económicos (OCDE) y la Oficina de Estadísticas de las Comunidades Europeas (EUROSTAT). (2006). Manual de Oslo. Guía para la recogida e interpretación de datos sobre innovación. 3ra. Edición.

Picón, Gilberto (1994). El Proceso de Convertirse en Universidad. Aprendizaje Organizacional en la Universidad Venezolana. Caracas; Fondo Editorial de la Universidad Pedagógica Experimental Libertador, Serie Investigaciones Educativas.

Von Krogh, Georg; Ichijo, Kazuo; Ikujiro, Nonaka (2001). Facilitar la Creación de Conocimiento. Cómo Desentrañar el Misterio del Conocimiento tácito y Liberar el Poder de la Innovación. Traducción: Enrique Cruz Mercado González. Oxford University Press. 